\title{
New Broadband Predistortion Linearizer Using Balanced Circuits Structure for Satellite TWTAs
}

\author{
Changwei Chen, Yong Cao and Kaiyu Qin \\ Institute of Astronautics and aeronautics Science and Technology, the University of Electronic Science and Technology of China
}

\begin{abstract}
The power amplifies is a key component influencing the power efficiency of communication system. The travelling wave tube is widely used in satellite system because of its high efficiency, apposite weight and broadband characteristic, but its strong nonlinear characteristic generates intermodulation distortion, which degrades the data quality of transmitted signal and causes interference in adjacent channels. The analogue predistortion structure using Schottky diodes could improve the linearity well, but parasite element in the Linearizer limits predistortion broadband, reluctant to satisfy the growing high volume data transmission requirement. This paper presents a new balanced structure of Linearizer for the travelling wave tube amplifier. We have analyzed the model of linearizer and the TWTA and used to calculate the intermodulation distortion and optimize the Linearizer nonlinear expansion characteristic. A frequency bandwidth of $1.2 \mathrm{GHz}$ Linearizer has been demonstrated with a K-band TWTA between 19.8 and $21.0 \mathrm{GHz}$. The measurement result shows that the linearizer delivers about $2 \sim 6 \mathrm{~dB}$ of gain expansion and 20 60 degrees phase shift expansion, and the cascaded LTWTA shows good linearity improvement of $17.8 \mathrm{~dB}$ at $6 \mathrm{~dB}$ input power back off point.
\end{abstract}

\section{Introduction}

With the rapid growth of high volume data in satellite communication systems, the modern millimeter wave satellite communication use non-constant envelope digital modulation techniques and demand for the wideband, high efficiency, and linear high-power amplifiers. The traveling wave tube amplifier is widely used in space application because of its high efficiency [1], but it has strong nonlinear characteristic. The nonlinearity generates intermodulation distortion (IMD), which causes interference in adjacent channels. To compensate these nonlinear distortions, many linearization techniques have been proposed, such as feedback, analog predistortion, feedforward, baseband digital predistortion. Among these technique such as baseband prediction, Practical DSP-based linearization systems of baseband predistortion can give a satisfactory IMD correction, but it is hard to reach bandwidth over $100 \mathrm{MHz}$ because of its complexity, cost, power consumption, and availability of required DSP components [2], [3]. The feedforward technique is too complex to implement on satellite.

Analogue predistortion technique has been the only choice in space applications because of their low power consumption, compactness, high reliability, and wide absolute bandwidth and easy to use.

There has been lots of analog predistortion structure proposed using Schottky diodes [4], and [5] or FETs [6] as the nonlinear device to produce the Inverse nonlinear distortion. It is relatively difficult to design a broad band
Linearizer with parasite element in the Linearizer. The Linearizer bandwidth in $[7,8]$ is only $400 \mathrm{MHz}$.In this paper, a broadband millimeter wave predistortion TWT linearizer is presented. The linearizer use Symmetrical structure, which expands the bandwidth and improves the linearizer input/output VSWR, and any additional isolator isn't required for matching.

\section{Linearizer characterize analysis}

\subsection{The analysis and compensation of the TWTA nonlinearity}

The TWTA is a memoryless and strong nonlinear amplifier, and the signal is treated as narrow band-limited signal, Let the input signal be

$$
x(t)=r(t) \cos \left[\omega_{0} t+\phi(t)\right]
$$

where $\omega_{0}$ is the carrier frequency, and $r(t)$ and $\psi(t)$ are the modulated envelope and phase, respectively. The corresponding output is written as

$$
y(t)=A[r(t)] \cos \left\{\omega_{0} t+\phi(t)+\Phi[r(t)]\right\}
$$

The output voltage can be expressed function of input signal with Saleh model [9] as eq. (2). The model presents nonlinear models of TWT amplifiers that are based on a simple two-parameter formula for each of the four aforementioned functions. For each of several cases 
examined these formulas fit TWT measurements accurately.

$$
\left\{\begin{array}{l}
A[r(t)]=\frac{\alpha_{a} r(t)}{1+\beta_{a} r^{2}(t)} \\
\Phi[r(t)]=\frac{\alpha_{\varphi} r^{2}(t)}{1+\beta_{\varphi} r^{2}(t)}
\end{array}\right.
$$

The Predistortion Linearizers are widely used in TWT amplifier linearization at microwave and higher frequencies. The Linearizer is cascaded before the TWT amplifier which provides the opposite nonlinear transfer response to TWT amplifier both in amplitude and phase characteristic as shown in Fig. 1. The gain expansion of the Linearizer compensates the gain expression of the TWT amplifier, and the phase increase of the Linearizer cancel the phase change of the TWT amplifier.

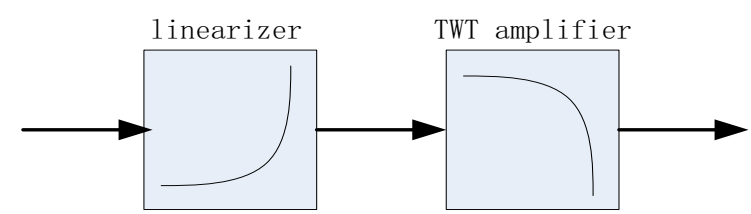

Figure 1. Predistortion Linearizer transfer characteristic schematic diagram

Using cascade net theory, the ideal AM-AM and AMPM characteristic of predistortion linearizer is deduced, the ideal predistortion linearizer model is as (4) and (5)

$$
\begin{gathered}
r(t)=\left\{\begin{array}{l}
\frac{\alpha_{a}-\sqrt{\alpha_{a}{ }^{2}-4 \beta_{a} \rho^{2}(t)}}{2 \beta_{a} \rho(t)}, 0<\rho(t) \leq G_{s} \\
G_{s}, G_{s} \leq \rho(t)<\infty
\end{array}\right. \\
\theta(t)=\frac{-\alpha_{\phi}\left(\alpha_{a}-\sqrt{\alpha_{a}^{2}-4 \beta_{a} \rho^{2}(t)}\right)^{2}}{4 \rho^{2}(t) \beta_{a}^{2}+\beta_{\phi}\left(\alpha_{a}-\sqrt{\alpha_{a}{ }^{2}-4 \beta_{a} \rho^{2}(t)}\right)^{2}} \\
0 \leq \rho(t) \leq \infty
\end{gathered}
$$

where, $G_{s}=\frac{1}{\sqrt{\beta_{a}}}, \rho(t)$ is the input voltage of the linearizer, $r(t)$ is the output voltage of the linearizer, $\theta(t)$ is the phase shift when the signal pass through the linearizer, $\alpha_{a}, \beta_{a}, \alpha_{\varphi}, \beta_{\varphi}$ is the parameter of the Saleh model.

For simplifying the IMD calculation, the nonlinear characteristics could be expressed as complex power series:

$$
v_{i n}=g_{1} A_{i n}+g_{3} A_{i n}^{3}+g_{5} A_{i n}^{5}
$$

Employing standard minimum mean-square-error curvefitting procedure, one obtains the required optimum values of $\mathrm{g} 1, \mathrm{~g} 2$ and $\mathrm{g} 3$ of the complex power series. Using the quadrature model of TWTA [6] calculate the IMD of the cascaded linearized TWTA, optimized the IMD and get the optmum inverse characteristic of TWTA as the design goal of the linearization design. The optimum value of Linearizer gain expansion and phase expansion is $3.8 \mathrm{~dB}$ and $37^{\circ}$. Accurate gain and phase expansion is required for satisfactory IMD correction in band. The IMD curve of the linearized TWTA showed as the Fig. 2 after optimum.

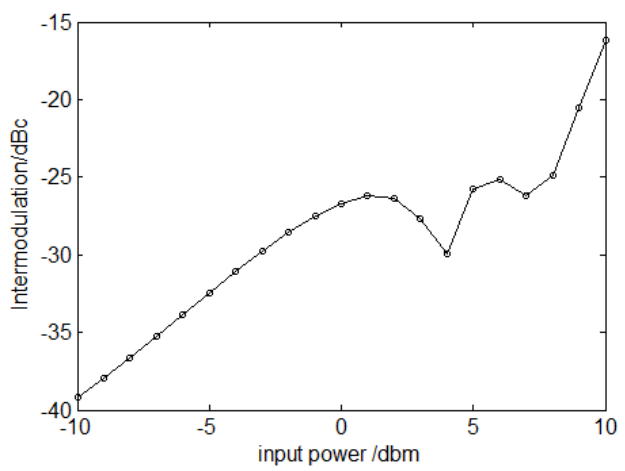

Figure 2.optimum IMD curve of the linearized TWTA.

\subsection{Reflective schottky diode predistorter using balanced structure}

The schematic diagram of the proposed predistortion Linearizer is shown in Fig. 3.

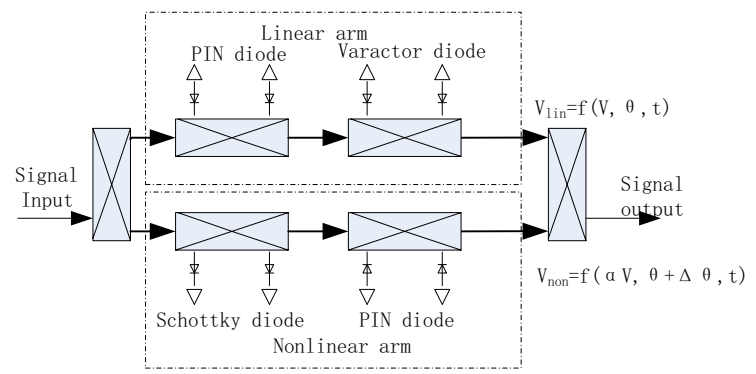

Figure 3. Predistortion Linearizer schematic diagram

The predistortion Linearizer is composed with linear arm and nonlinear arm with symmetrical structure. The nonlinear arm is constructed by a $3 \mathrm{~dB}$ bridge with a Schottky barrier diode, which generated nonlinear component. The linear arm is constructed by attenuator and phase shifter, which match the gain and phase shift between the linear arm and nonlinear arm. These two arms are connected with a $3 \mathrm{~dB}$ branch line coupler input power divider and a $3 \mathrm{~dB}$ branch line coupler output power divider. This Symmetrical structure improves the bandwidth and VSWR of the Linearizer.

In the predistortion Linearizer schematic diagram, the $v_{L I N}$ is the output signal from the linear arm, it is a function of the input amplitude, phase and the group delay. The linear arm output signal could be written

$$
V_{L I N}=f(v, \theta, t)=V[\cos (\omega t+\theta)]
$$

The signal $v_{N O N}$ is the output signal from the nonlinear arm. After the adjust of the amplitude and phase, it could be written as

$$
V_{N O N}=f(\alpha v, \theta+\Delta \theta, t+\Delta t)
$$




$$
=\alpha V[\cos (\omega t+\theta+\Delta \theta)]
$$

where, the variable $\alpha$ is amplitude ratio between the linear arm and nonlinear arm. It is decided by the nonlinear generator which is composed of schottky diode, and attenuator composed of PIN diode. It is a function of input signal amplitude.

The variable $\Delta \theta$ is phase difference between the linear arm and nonlinear arm. It is decided by the nonlinear generator and phase shifter. It is a function of input signal amplitude.

When the two signals are combined, the result is

$$
V_{\text {out }}=V[\cos (\omega t+\theta)+\alpha \cos (\omega t+\theta+\Delta \theta)]
$$

Using the trigonometric function transformation, the formulas (8) could be converted as

$$
V_{\text {out }}=g V[\cos (\omega t+\theta+\phi)]
$$

where,

$$
\begin{gathered}
g=\sqrt{1+\alpha^{2}+2 \alpha \cos \Delta \theta} \\
\phi=\arctan \frac{\alpha \sin \Delta \theta}{1-\alpha \cos \Delta \theta}
\end{gathered}
$$

The vector diagram of Fig. 4 illustrates the subtracting a linear arm signal from a nonlinear arm signal at high and low amplitude level. The gain of the nonlinear arm $V_{N O N}$ decreases as the input power increases. Appropriate adjustment of the amplitude ratio $\alpha$ and angle difference $\Delta \theta$ between the linear and nonlinear arm allows the output signal level change in positive direction same as required expansion transfer function.

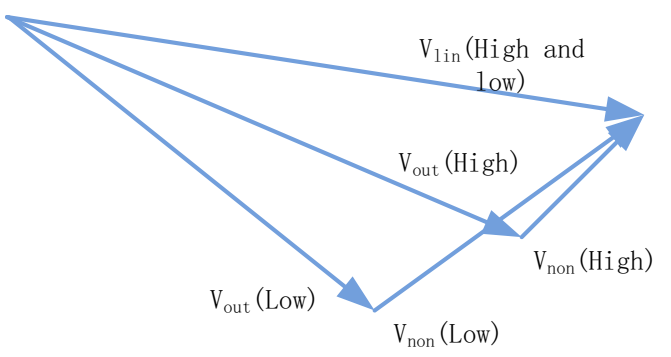

Figure 4. Anoalogue predistortion of subtracting a linear path from a nonlinear path

In the section 2.1, satisfactory broad bandwidth IMD correction needs consistent gain and phase expansion in band. From the formula (10) to (12), the output signal gain and phase is associated with the amplitude ratio $\alpha$ and angle difference $\Delta \theta$ between the linear and nonlinear arm, regardless of the flatness of the linear and nonlinear arm.

Parasite element in the Linearizer deteriorates the gain and phase flatness of Linearizer linear or nonlinear arm. The symmetrical structure of the Linearizer could be used to keep the amplitude ratio $\alpha$ and angle difference $\Delta \theta$ consistent in the band under influence of the parasite element in the Linearizer.
The photograph of the manufactured Linearizer module is shown in Fig. 5. The Linearizer is realized in a very compact size of $18 \times 6 \mathrm{mmm}$ and its substrate used is alumina with the dielectric constant of 9.9 and thickness of $0.254 \mathrm{~mm}$. The used schottky diode is HSCH-5332 of Agilent. The PIN diode used in attenuator is HPND4005 of skyworks. The Silicon hyperabrupt junction varactors diode used in phase shifter, which has about $60^{\circ}$ adjust range, is SMV2019 of skyworks.

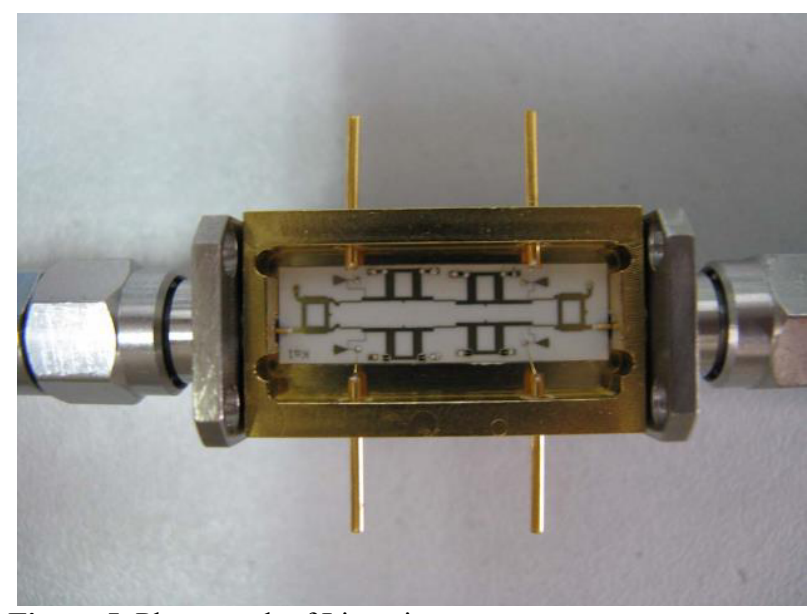

Figure 5. Photograph of Linearizer

\section{Experimental results}

The AM-to-AM and AM-to-PM of the calculated above is realized, and could be tuned with adjusting the bias the control voltage of the attenuator and the phase shifter. The shape tuning capability [8] could be gotten through the bias with the control voltage of the nonlinear generator. The AM-to-AM and AM-to-PM expanding nonlinear characteristic matched with TWTA nonlinear curve is a key to the linearization.

The AM-to-AM and AM-to-PM expanding nonlinear characteristic has been tested using Agilent Technologies' PNX series large signal network analyzer N5244, the AM-to-AM and AM-to-PM curve result figure is shown in Fig. 6. The measurement result shows that the Linearizer delivers about $2-6 \mathrm{~dB}$ of gain expansion and 20-60 degrees phase shift expansion characteristic and could be adjustable and be consistent in band.

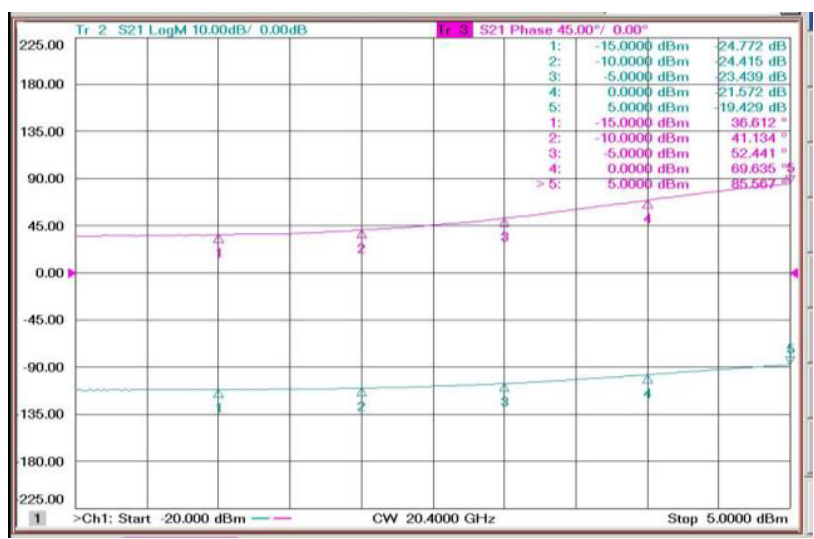

Figure 6.Gain expansion and phase expansion of Linearizer 
The output power transfer characteristic of the TWTA with and without lineariztion has been tested using Agilent Technologies' signal generator and power meter the curve result figure is shown in Fig. 7. The power sweep from $0 \mathrm{~dB}$ IBO (input power back off) to $20 \mathrm{~dB}$ IBO. The output power transfer characteristics figure shows the good performance of the Linearizer.

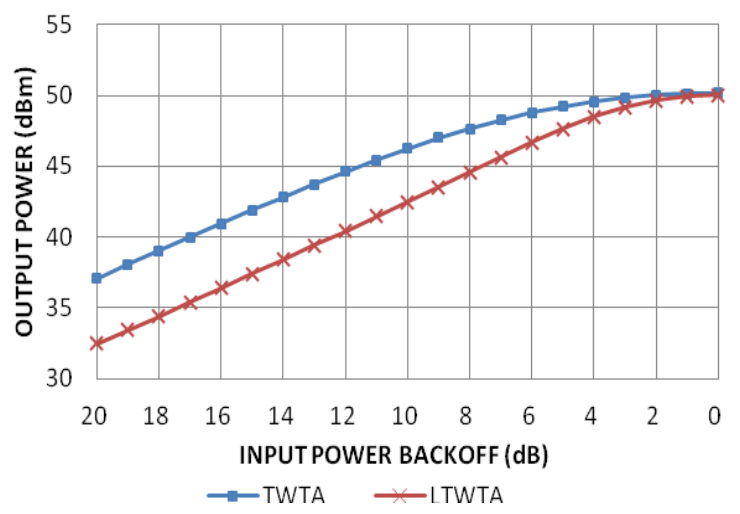

Figure 7. Output power transfer characteristics of the TWTA with and without lineariztion

The IMD of the linearised TWT amplifier has been tested using Agilent Technologies' PNX series large signal network analyzer N5244, Two-tone IMD performance of TWT amplifier with TWTA and TWTA with predistorter and feedforward amplifier was tested separately. The tone spacing is selecting to be $5 \mathrm{MHz}$. The IMD result figure is shown in Fig. 8.

The performance of the Linearizer is evaluated with a $100 \mathrm{~W}$ K-band TWTA between 19.8 and 21.0GHz. An amplifier CHA3689 and an attenuator CHA3091 of UMS are used between the Linearizer and the TWTA is used to compensated the loss of Linearizer and adjust the TWT required input power.

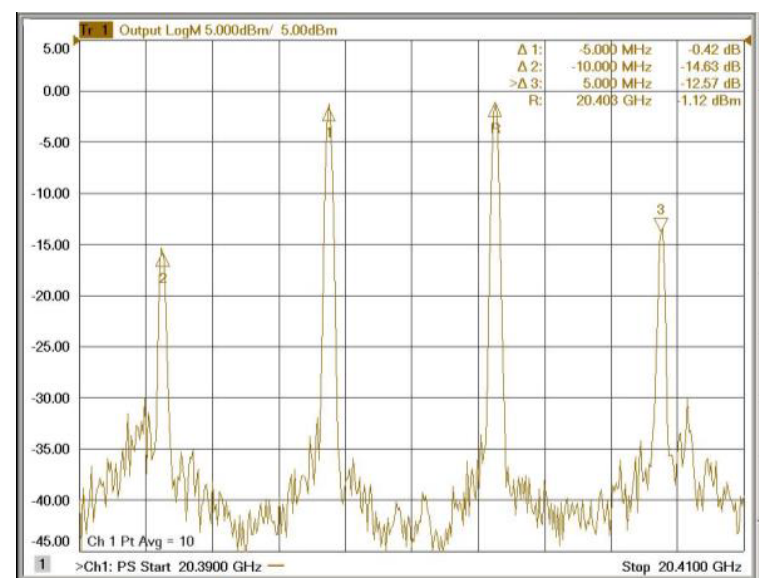

Figure 8. IMD of the LTWTA working at saturation point

The cascade linear TWTA gain compression is compensated to $0.8 \mathrm{~dB}$. The phase shift compression is compensated to $-5.8^{\circ}$ at the input saturation point. The IMD performance of the LTWTA is improved, which
$2.1 \mathrm{~dB}$ at $0 \mathrm{~dB} \mathrm{IBO}$, and $17.8 \mathrm{~dB}$ improvement at $6.5 \mathrm{~dB}$ IBO which is improvement optimum point as shown in Fig. 9.

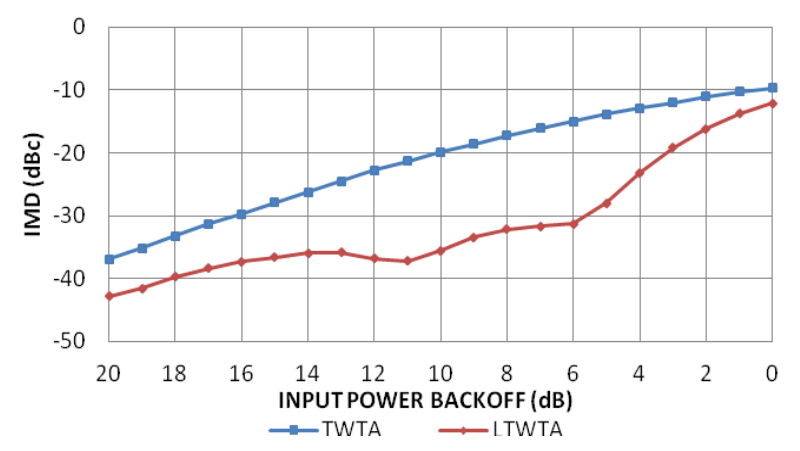

Figure 9. The IMD performance of the TWTA and linearised TWTA

\section{Conclusions}

The model of the nonlinear TWT and Linearizer is established and predict the optimum value of Linearizer gain expansion and phase expansion with quadrature model of TWTA. With the optimum value of Linearizer, A two-way linearizer signal subtraction is analyzed and gives the condition of accurate nonlinear gain and phase expansion. Using balance structure of linearizer, the parasite element influence is cancelled and extends the bandwidth of the linearizer. Good input and output VSWR characteristic make it connect to amplifier without isolator due to the symmetrical structure. The cascaded LTWTA shows good nonlinearity improvement of $2.1 \mathrm{~dB}$ at $0 \mathrm{~dB}$ IBO and $17.8 \mathrm{~dB}$ at $6.5 \mathrm{~dB}$ IBO.

\section{References}

1. E. Cuignet, E. Tonello, J. Maynard, P. Boone, IVEC2013, 14th ,Pag: 1 - 2(2013).

2. A. Katz, R. Gray, R. Dorval, IEEE micro. Mag., J.10, 7, pp. 20 27 (2009).

3. X. Feng, B. Feuvrie, A.S. Descamps, Y. Wang, Electr. Lett., J., 50, (24), pp. 1882-1884(2014).

4. A. Katz, R. Gray, R. Dorval, IEEE Trans. Electr. Dev., 56, 5, pp. 959 - 964 (2009).

5. A. Katz, R. Sudarsanam, C. Aubert, MTTS Int. Microw. Symp. Dig., pp. 661-664 (1985).

6. A. Katz, R. Domal, IEEE MTT-S Digest, pp. 437440 (1994)

7. H.Y. Jeong, S.K. Park, N.S. Ryu, Y.C. Jeong, $E G A A S$, pp. 597-600(2005).

8. S.C .Bera, V. Kumar, S. Singh, D.K. Das, 23, 4, pp. $211-213(2013)$.

9. A. A. M. Saleh, Tans. on Comm., 29, 11, pp. 17151720. (1981). 